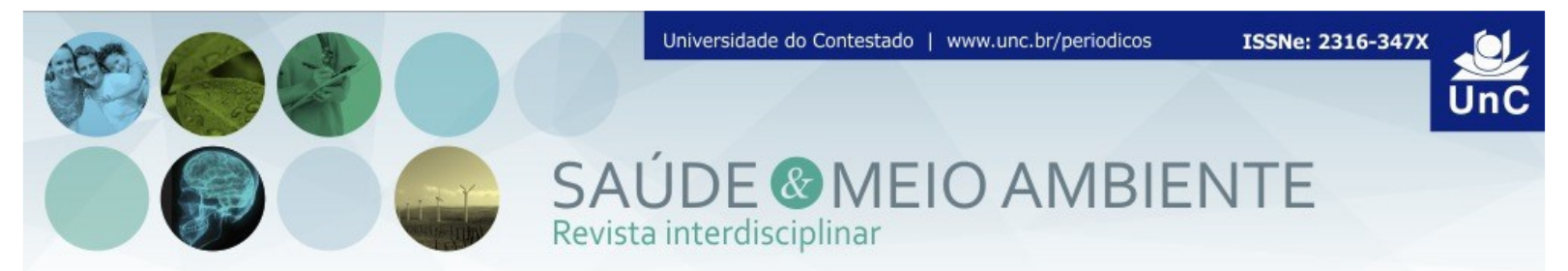

\title{
A MÚSICA COMO PRÁTICA INTEGRATIVA COMPLEMENTAR EM IDOSOS INSTITUCIONALIZADOS ${ }^{1}$
}

\section{MUSIC AS A INTEGRATIVE AND COMPLEMENTARY PRACTICE IN INSTITUTIONALIZED ELDERLY}

Thaynara Sabrine Pscheidt ${ }^{2}$

Paty Aparecida Pereira ${ }^{3}$

\begin{abstract}
RESUMO
INTRODUÇÃO: A população passou por um processo de envelhecimento em um curto prazo de tempo, devido ao aumento da expectativa média de vida e as quedas significativas nas taxas de fecundidade e mortalidade dos últimos anos. Esses fatores, aumentam o comprometimento desta população pelas doenças crônicodegenerativas e pelas Incapacidades Funcionais (IFs), motivos que os levam a institucionalização. Em busca da qualidade de vida desses idosos, as instituições de longa permanência vêm desfrutando do benefício da musicoterapia, cuja terapia busca prevenir e minimizar os sintomas de várias doenças. OBJETIVOS: Analisar os efeitos terapêuticos da música na promoção de saúde em idosos de uma instituição de longa permanência. MATERIAIS E MÉTODOS: Tratou-se de uma pesquisa do tipo qualitativa, com características de pesquisa-ação, de forma exploratória e intervencionista. Foi realizado em uma Instituição de Longa Permanência de uma cidade do Planalto Norte Catarinense. Contou com uma amostra de 5 participantes. $\mathrm{Na}$ avaliação foram aplicados os instrumentos MEEM e WHOQOL-OLD, os mesmos foram aplicados pré e pós intervenção, totalizando 10 encontros com duração de 45 minutos. Ao termino foi aplicado um questionário de satisfação dos participantes. RESULTADOS: O MEEM obteve aumento na avaliação pré e pós 10 encontros. Na avaliação do WHOQOL-OLD, foi observado aumento pós intervenção nos domínios de autonomia, atividades passadas, presentes e futuras e no domínio de intimidade. A satisfação dos pacientes em participar da pesquisa foi positiva. CONCLUSÕES: A musicoterapia apresenta resultados positivos para melhora do cognitivo, qualidade de vida, e a socialização dos idosos de uma ILP.
\end{abstract}

Palavras-Chave: Envelhecimento. Musicoterapia. Qualidade de vida.

\footnotetext{
${ }^{1} \mathrm{O}$ presente trabalho foi realizado com apoio do Programa de bolsas Universitárias do Estado de Santa Catarina - UNIEDU

${ }^{2}$ Acadêmica do Curso de Fisioterapia. Universidade do Contestado. Mafra. Santa Catarina. Brasil. Email: thaynara.sabrine@hotmail.com

${ }^{3}$ Mestre em Tecnologia em Saúde (PUC-PR). Especialista Fisioterapia Respiratória e Cardiovascular Centro Brasileiro de Estudos Sistêmicos (CBES). Fisioterapeuta - Graduada pela Universidade Positivo. Docente da Universidade do Contestado, Mafra, SC. Brasil. E-mail: fisio.paty@gmail.com. ORCID https://orcid.org/0000-0002-1909-3441
} 


\begin{abstract}
INTRODUCTION: Population passed through an aging process in a short time, because the increased life expectancy and because the significant declines in fertility and mortality rates in recent years. These factors increase the involvement of this population with chronic degenerative diseases and Functional Disabilities (Incapacidades Funcionais - IFs), reasons that lead them to institutionalization. Searching for the life quality of the elderlies, long-term care institutions have been enjoying the benefit of music therapy, which seeks to prevent and minimize the symptoms of various diseases. OBJECTIVE: To analyze the therapeutic effects of music on elderly health promotion of a long-term care institution. MATERIALS AND METHODS: This was a qualitative research, with characteristics of action research, exploratory and interventionist. The research was developed in a long-term care Institution of a city in the Planalto Norte Catarinense (Northern Santa Catarina Plateau) and had a sample of 5 participants. The MEEM and WHOQOL-OLD instruments were applied before and after the intervention, which totaled 10 meetings lasting 45 minutes each At the end the participants answered a satisfaction questionnaire. RESULTS: After 10 meetings, MEEM increased. In the WHOQOLOLD evaluation, an increase was observed after intervention in the domains of autonomy, past, present and future activities and in the domain of intimacy. Satisfaction of the research patients was positive regarding participation. CONCLUSIONS: Music therapy has positive results for cognitive improvement, quality of life, and socialization of the elderlies who live in a Long-term care institution.
\end{abstract}

Keywords: Aging. Music therapy. Quality of life.

\title{
1 INTRODUÇÃO
}

A população idosa vem apontando mudanças na estrutura etária da sociedade mundial. Segundo as estatísticas da Organização das Nações Unidas (ONU), ${ }^{1}$ até 2025 , o mundo terá 840 milhões de pessoas idosas, o que representa $70 \%$ de pessoas na $3^{\mathrm{a}}$ idade e o Brasil neste mesmo ano, será o $6^{\circ}$ maior país em população de idosos. O Instituto Brasileiro de Geografia e Estatística (IBGE), ${ }^{2}$ estima que um quarto da população do país deverá ter mais que 65 anos em 2060.

Pode-se atribuir isso, a fatores como o aumento da expectativa média de vida da população e as quedas significativas nas taxas de fecundidade e mortalidade dos últimos anos, que foi essencial para o processo de envelhecimento acontecer em um curto prazo de tempo ${ }^{3}$.

Atualmente, com o aumento da expectativa de vida e o estilo sedentário da população, a qualidade de vida dos idosos foi comprometida pelas doenças crônicodegenerativas e pelas Incapacidades Funcionais (IFs), acarretando um maior número de hospitalizações desses indivíduos ${ }^{4}$. Diante desses fatos, o campo de estudo sobre geriatria e gerontologia reforça pesquisas de intervenções 
terapêuticas, preventivas e da reabilitação, promovendo o envelhecimento ativo e funcional, consequentemente, melhorando as condições de vida e saúde desta população ${ }^{5}$.

No envelhecer, o organismo do ser humano passa por modificações funcionais e estruturais, que reduzem a vitalidade e favorecem o aparecimento de doenças crônicas não transmissíveis. Para que o idoso viva sua longevidade da melhor maneira possível, ou seja, viver com qualidade, deve-se ter um cuidado especial nesta fase da vida ${ }^{6}$.

Segundo Nunes, Ferratti e Santos, ${ }^{7}$ ser velho é um acontecimento inevitável, onde todos os seres humanos estão sujeitos com a passagem do tempo e este processo ocorre de maneira gradual, ativa e irreversível, com várias mudanças genético-biológicas, psicossociais e fisiopatológicas. Barbosa et $\mathrm{al}^{8}$, associam ao envelhecimento: Alterações no sistema nervoso, somatossensorial, musculoesquelético, cardiorrespiratório, visual, vestibular e mudanças cognitivas, e que, com o uso de medicamentos e pela presença de doenças crônicas, as alterações desses sistemas podem ser agravadas. Fatores que para Saldanha e Caldas, ${ }^{9}$ impõem limitações de mobilidade e independência, prejudicando a interação social, a realização de atividades e comprometendo a qualidade de vida do idoso.

As perdas funcionais, doenças, sedentarismo, níveis de saúde precários, condições socioeconômicas desfavoráveis, assistência familiar, seja financeira ou psicológica insatisfatória e falta de cuidador são os principais motivos que levam a institucionalização ${ }^{10}$.

A instituição de longa permanência torna o idoso isolado da sociedade, ocasionando a diminuição de sua autoestima, causando a perda da identidade e por consequência, fazendo com que o idoso se sinta desvalorizado. Ribeiro e Schutz, ${ }^{11}$ mencionam que os idosos que vivem em uma instituição de longa permanência, longe do convívio familiar, apresentam declínio na saúde, pois têm como consequência $o$ isolamento, a inatividade física e mental, comprometendo a qualidade de vida do indivíduo. Para Candeias ${ }^{12}$, esses idosos são forçados a adaptar-se as novas rotinas, novas pessoas, adequar-se ao ambiente, do qual não são os donos, e, contudo, não possuem o poder das decisões, tanto da casa, quanto das suas próprias vidas, mudanças que afetam significativamente a qualidade de vida desses idosos, tornando-os mais vulneráveis a doenças. Com isso, é essencial a realização de atividades físicas, que promovam o bem-estar físico e mental dos idosos de uma instituição de longa permanência.

Nesta perspectiva observa-se na atualidade o surgimento de pesquisas sobre novas intervenções terapêuticas, preventivas e de reabilitação no intuito de promover o envelhecimento ativo e funcional, proporcionando, por conseguinte, qualidade de vida. Estas investigações vão ao encontro da Política Nacional de Saúde da Pessoa Idosa (PNSPI) a qual foi aprovado em 19 de outubro de 2006, através da portaria $n^{\circ}$ 2.528. A PNSPI tem por finalidade recuperar, manter $\mathrm{e}$ promover a autonomia e a independência do idoso, através de medidas coletivas e individuais de saúde ${ }^{13}$. 
Em busca da qualidade de vida dos idosos, as instituições de longa permanência vêm desfrutando do benefício da música como tratamentos terapêuticos. A musicoterapia é uma intervenção barata, agradável, sem efeitos adversos ${ }^{14}$ e tem um papel relevante no trabalho com idosos, contribuindo assim para as políticas de promoção e prevenção da saúde, a fim de melhorar a expectativa e a qualidade de vida dessa população ${ }^{15}$. Ela é considerada uma terapia autoexpressiva, capaz de estimular o físico, o emocional e proporcionar uma melhorara na vida social desses idosos. Também possui forte atuação na prevenção de doenças, onde avigora o potencial criativo do idoso, a sua socialização e fortalece a sua memória ${ }^{16}$.

Atividades musicais regulares são muito promissoras, melhorando o humor e impactando positivamente na qualidade de vida e no bem-estar dos idosos, promovendo empoderamento, autonomia e coesão social dos mesmos ${ }^{17,18}$. Rossetto afirma que a Musicoterapia melhora a integração do idoso com a sua própria saúde e com o ambiente em que ele vive diariamente ${ }^{19}$.

Tendo em vista toda essa problemática, entende-se que este estudo é relevante e que existem incipientes pesquisas relacionadas à utilização da música como terapia para idosos que vivem em uma instituição de longa permanência.

Sendo assim, este estudo busca mostrar os efeitos terapêuticos da música na promoção de saúde em idosos de uma instituição de longa permanência.

\section{MATERIAIS E MÉTODOS}

O presente estudo teve uma abordagem qualitativa, com características de pesquisa-ação, de forma exploratória e intervencionista. Foi realizado em uma Instituição de Longa Permanência de uma cidade do Planalto Norte Catarinense.

A população estudada foi composta por 10 idosos que aceitaram participar da pesquisa, com idade igual ou superior a sessenta anos, que estivessem residindo na instituição pelo menos há dois meses. Sendo excluídos os que não tiveram condições de expressar reações mensuráveis às situações avaliadas na pesquisa, os que não obtiveram assiduidade nas práticas realizadas (frequência menor que 70 \%) e os que não assinaram o Termo de Consentimento Livre e Esclarecido.

Esse estudo foi submetido ao Comitê de Ética em Pesquisa da Universidade do Contestado Campus Mafra-SC, realizada respeitando os princípios éticos contidos na Resolução $N^{\circ} 466 / 12$, do Conselho Nacional de Saúde (CNS), que trata de pesquisas envolvendo seres humanos ${ }^{20}$, aprovado sob número do Parecer 3.277.065.

Os participantes foram esclarecidos sobre os objetivos da pesquisa, o caráter de anonimato, assim como o direito de desistência a qualquer momento durante a realização da mesma sem nenhum questionamento contra a decisão do participante. 
A coleta de dados foi realizada pelas próprias pesquisadoras, inicialmente, obtido dados sócio demográficos dos idosos, através da coleta de dados de prontuários (idade, gênero, tempo de internação, escolaridade, estado civil, raça e o motivo da institucionalização). Após, aplicado dois instrumentos com o intuito de avaliar capacidade cognitiva e qualidade de vida. Avaliamos a capacidade cognitiva geral aplicando o MEEM - Mini exame do Estado Mental ${ }^{21}$, um teste cognitivo breve composto por itens a respeito de orientação temporoespacial, registro, memória de curto prazo, atenção, calculo, linguagem e praxia construcional. No que diz respeito aos aspectos relacionados à orientação temporal, os idosos foram questionados sobre o dia, mês e ano; em relação à orientação espacial, sobre a cidade, o bairro e o local que estavam no momento da aplicação do MEEM. Quanto ao registro, os indivíduos foram instruídos a repetir três palavras: "casa", "vaso" e "tijolo". Para o item cálculo, foram propostos números para subtração. Na categoria memória, os idosos tiveram que evocar as palavras repetidas no item registro. A respeito da linguagem, os sujeitos nomearam os objetos, caneta e relógio, e repetiram a frase "nem aqui, nem ali, nem lá", e realizaram a cópia de um desenho com dois pentágonos em interseção.

Ressalta-se que em cada categoria é indicada uma determinada pontuação máxima, sendo que no total o indivíduo pode somar 30 pontos e conforme o ponto de corte (24 pontos), consideram-se as funções cognitivas adequadas ou não, até mesmo é possível identificar possíveis alterações ${ }^{22}$.

A qualidade de vida da amostra foi avaliada por meio do questionário WHOQOL-OLD, que contém seis facetas (ou domínios) de quatro itens cada (o módulo consiste em 24 itens), avaliados pela escala de Likert (1 a 5 pontos) atribuídos para cada faceta. Para todas as facetas, o escore dos valores possíveis pode oscilar de 4 a 20 , desde que todos os itens de uma faceta tenham sido preenchidos. Os escores destas seis facetas ou os valores dos 24 itens do módulo WHOQOL-OLD, podem ser combinados para produzir um escore geral (QVG-OLD) para a qualidade de vida em adultos idosos, denotado como o "escore total" do módulo WHOQOL- OLD ${ }^{23}$. Basicamente, escores altos representam uma alta qualidade de vida e escores baixos representam uma baixa qualidade de vida.

Aplicamos o questionário individualmente, solicitamos aos participantes que ao responder tivessem em mente os próprios valores, aspirações, prazeres e preocupações, tomando por base as duas últimas semanas.

A intervenção proposta contou com 12 encontros, os mesmos aconteceram 1 vez por semana. O primeiro e o último encontro foram utilizados para realizar as avaliações. No primeiro apresentamos como iria acontecer a atividade, coletando informações dos dados sociodemográficos, e, neste momento também foi questionado ao idoso quais músicas seriam de sua preferência, e na sequencia realizada a aplicação dos questionários, sendo estes, aplicados novamente no $12^{\circ}$ encontro. Antes de dar início a intervenção, junto com o responsável da instituição, foram previamente agendadas as datas em que aconteceram as atividades.

Cada sessão teve duração de 45 minutos, realizadas em grupo, onde os participantes sentaram em cadeiras dispostas em um círculo e no primeiro momento 
da dinâmica foi chamado de "Identidade", com a finalidade de apresentação pessoal de cada participante. Perguntamos a cada idoso seu nome e idade, que foi repetida em voz alta para que todos ouvissem, contribuindo desta forma, para maior interação entre todos e melhor desenvolvimento da dinâmica seguinte.

Antes e ao término de cada sessão de musicoterapia, avaliamos a pressão arterial do participante e realizamos um aquecimento e relaxamento. Foram realizados movimentos circulares e de flexão e extensão das articulações de membros superiores e membros inferiores, e, exercícios respiratórios com inspirações e expirações lentas e profundas. Dentro das atividades, pudemos estimular a socialização, o desenvolvimento da percepção rítmico-sonora, da expressividade, da memorização, da concentração, da confiança e do otimismo.

Após realizado as etapas iniciais, começamos a atividade com as músicas. Utilizamos 8 músicas em cada sessão, considerando em média 4 minutos cada uma. As músicas escolhidas para prática, foram relacionadas com a preferência musical dos participantes, com base nos dados obtidos na avaliação inicial. Os participantes cantaram, dançaram em bipedestação ou sentado conforme estavam sentindo-se mais confortáveis e seguros no momento, os mesmos eram estimulados em realizar as atividades através do comando verbal das pesquisadoras. Foram utilizados nas sessões aparelho de som com CD, violão, pandeiros, chocalhos, bola e balões, permitindo sempre que os idosos interagissem ativamente.

Ao final da intervenção, além da música, da aplicação do questionário MEEM e WHOQOL-OLD, foi aplicado também um outro questionário elaborado pelas pesquisadoras, com o objetivo de avaliar a satisfação dos idosos, semiestruturado com 7 perguntas, com alternativas de respostas fechadas em uma escala Likert de 5 pontos, na qual o valor 1 , indicava o menor grau de satisfação e o valor 5 , maior grau de satisfação da terapia.

Quadro 1 - Questionário semiestruturado de satisfação.

\begin{tabular}{|l|}
\hline \multicolumn{1}{|c|}{ PERGUNTA } \\
\hline 1 - Você gostou da atividade? \\
\hline 2 - As explicações oferecidas pelo fisioterapeuta foram realizadas com clareza? \\
\hline 3 - O fisioterapeuta teve gentileza nos atendimentos? \\
\hline 4 - As músicas foram...? \\
\hline 5 - Você gostaria que está atividade tivesse continuidade? \\
\hline 6 - Você considera que a atividade te trouxe mais qualidade de vida? \\
\hline 7 - Você gostaria que está atividade fosse implantada na rotina do lar São Francisco de Assis? \\
\hline
\end{tabular}

Fonte: Dados da pesquisa (2019)

Para análise dos resultados desta pesquisa foi utilizado software SPSS 10.0; através de estatísticas descritivas como frequência, média, desvio-padrão e teste t. 


\section{RESULTADOS}

Esta pesquisa foi realizada em uma Instituição de Longa Permanência, no período de abril a agosto de 2019. Inicialmente a amostra contou com a presença de 10 idosos, mas houve a desistência de 5 destes, alegando não gostarem de música. Os idosos participantes em questão, foram 5 indivíduos, tendo predomínio de mulheres (80\%), apresentando uma média de idade de $78 \pm 9,74$ anos. O tempo em que residiam na instituição foi de 4 anos o mínimo, e 13 anos o mais longo, destes, $40 \%$ eram analfabetos e $20 \%$ de baixa escolaridade, não concluíram o primeiro grau.

Com relação a capacidade cognitiva geral, foi analisado individualmente as estruturas do MEEM, onde observando a tabela 1, pode-se perceber que não houve diferença significativa na correlação pré e pós intervenção de musicoterapia entre as estruturas, porém, analisando a média geral, o MEEM obteve média de 17 pontos na avaliação pré intervenção, aumentando o escore médio para 20 na avaliação final. Com isso pode-se observar uma melhora no cognitivo dos participantes. Os dados estão apresentados na tabela 1.

Tabela 1 - Pontuação do MEEM, média e desvio padrão, pré e pós a intervenção com musicoterapia.

\begin{tabular}{lccc}
\hline & Média Pré & Média Pós & $\begin{array}{c}\text { P-valor } \\
(\leq \mathbf{0 , 0 5})\end{array}$ \\
\hline Orientação Temporal & $2 \pm 1,49$ & $3 \pm 0,48$ & 0,11 \\
\hline Orientação Espacial & $5 \pm 1,95$ & $4 \pm 1,35$ & 0,50 \\
\hline Memória Imediata & $3 \pm 0,40$ & $3 \pm 00$ & 0,18 \\
\hline Atenção e Calculo & $1 \pm 0,48$ & $0 \pm 0,80$ & 0,50 \\
\hline Memória Recente & $2 \pm 1,20$ & $2 \pm 0,63$ & 0,10 \\
\hline Linguagem & $6 \pm 0,74$ & $6 \pm 1,20$ & 0,08 \\
MEEM geral & $17 \pm 4,80$ & $20 \pm 2,78$ & 0,06 \\
\hline
\end{tabular}

Fonte: Dados da pesquisa (2019).

Para análise da qualidade de vida dos idosos, observamos que não obtivemos resultados positivos para qualidade de vida geral, ou seja, ao analisar a pontuação de todos os domínios individualmente. O WHOQOL-OLD obteve média de qualidade de vida geral de 72 pontos na avaliação pré intervenção da terapia com música e média de 64 pontos na avaliação pós intervenção. Observando a tabela 2, percebe-se a diminuição da qualidade de vida geral, porém, é fundamental ressaltar, que os domínios de autonomia, atividades passadas, presentes e futuras e domínio de intimidade, os quais estão relacionados com a intervenção proposta, e que foram estimulados durante a terapia, observamos resultados positivos e melhores, com aumento dos escores pós intervenção, como demonstra a tabela 2. 
Tabela 2 - Pontuação do WHOQOL-OLD, média e desvio padrão, pré e pós a intervenção com musicoterapia.

\begin{tabular}{lccc}
\hline Domínios & Média Pré & Média Pós & $\begin{array}{c}\text { P-valor } \\
(\leq \mathbf{0 , 0 5})\end{array}$ \\
Dom 1 & $15 \pm 4,14$ & $9 \pm 4,95$ & 0,11 \\
Dom 2 & $9 \pm 4,19$ & $11 \pm 3,96$ & 0,44 \\
Dom 3 & $10 \pm 5,03$ & $8 \pm 4,66$ & 0,28 \\
Dom 4 & $12 \pm 2,96$ & $14 \pm 2,87$ & 0,05 \\
Dom 5 & $16 \pm 2,57$ & $15 \pm 2,56$ & 0,18 \\
Dom 6 & $11 \pm 4,60$ & $14 \pm 3,76$ & 0,45 \\
QV Geral & $72 \pm 18,61$ & $64 \pm 17,19$ & 0,22 \\
\hline
\end{tabular}

Fonte: Dados da pesquisa (2019). Dom 1= Domínio Habilidades Sensoriais, Dom 2=Domínio Autonomia, Dom 3=Domínio Morte e Morrer, Dom 4=Domínio Atividades passadas, presentes e futuras, Dom 5=Domínio Participação Social, Dom 6=Domínio Intimidade, QV geral= Qualidade de vida geral.

A satisfação dos idosos com a intervenção musicoterapêutica é demostrada no gráfico 1 , onde percebe-se que $100 \%$ da amostra gostaria que esta atividade tivesse continuidade.

Gráfico 1 - Dados relativos a satisfação da participação na intervenção.

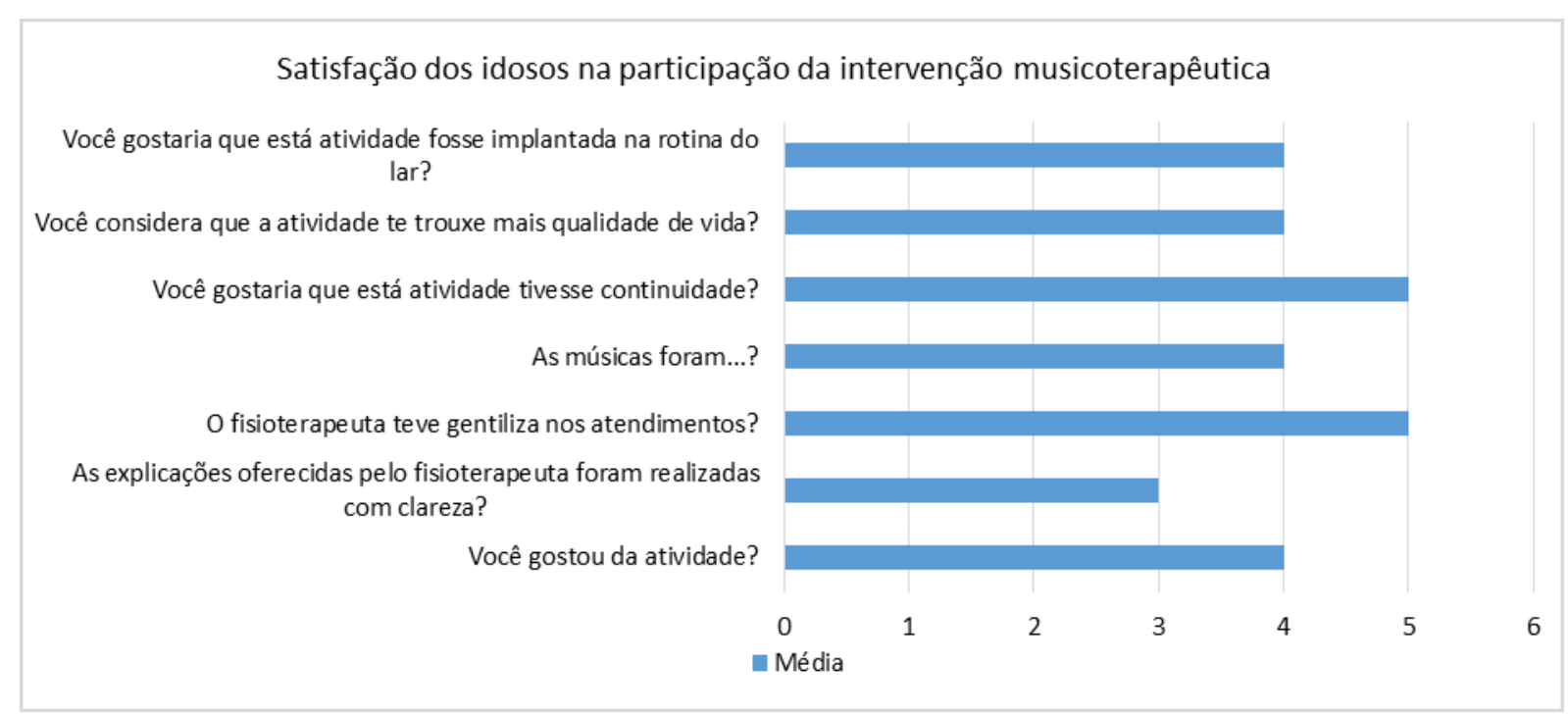

Fonte: Dados da pesquisa (2019).

\section{DISCUSSÃO}

Um estudo divulgado por Machado e colaboradores ${ }^{24}$, aponta que "idosos com um ano de estudo ou menos, tem 3,83 vezes mais chances de ter declínio cognitivo, do que os que tem de um a quatro anos de estudos". Com essa afirmação 
é possível compreender que os escores do presente estudo foram influenciados pelo baixo cognitivo dos idosos participantes, interferindo assim indiretamente nos resultados.

Segundo Fostein et al., ${ }^{21}$ o escore máximo do MEEM é 30 pontos para alfabetizados. Analisando o estudo de Lourenço e Veras, ${ }^{25}$ verificamos que o escore para analfabetos é menor, pois eles concluem que, para o rastreamento cognitivo de populações idosas, com ausência e/ou baixa escolaridade, deve-se considerar os pontos de corte 18/19. Andrade, Souza e Ferrer, ${ }^{26}$ corroboram com essa afirmação, apontando o nível educacional como forte influência na pontuação do MEEM, pois, em seu estudo composto por 80 idosos residentes de uma instituição, foram observados $80 \%$ de analfabetos com pontuação $\leq 19$ pontos e $66,7 \%$ idosos com nota de corte $\leq 23$ pontos com alguma escolaridade. Correlacionando o estudo com os demais citados, podemos considerar então, que os resultados encontrados neste, foram melhores no pós intervenção com a musicoterapia.

$\mathrm{Na}$ análise da qualidade de vida, os resultados mostram que os idosos avaliados, apresentam qualidade de vida inferior nas facetas habilidades sensoriais, morte e morrer e domínio de participação social, e, melhor qualidade para as facetas: autonomia, atividades passadas, presentes e futuras e intimidade, o que diverge do estudo de Serbim e Figueiredo ${ }^{27}$, onde os domínios que mais contribuíram para a qualidade de vida foram as facetas que não possuíram pontuação positiva no presente estudo.

Segundo Vieira, ${ }^{28}$ a faceta a autonomia está associada à independência dos idosos, a capacidade de viverem de forma autônoma e de tomarem suas próprias decisões. O aumento da faceta de autonomia, contribui para a maior satisfação dos idosos na instituição de longa permanência, em relação a tomarem suas próprias decisões ou fazer as coisas que gostam, e em seu estudo percebe-se, que a autonomia dos idosos reflete diretamente na qualidade de vida dos mesmos.

As facetas de atividades passadas, presentes e futuras são descritas por Miranda e Banhato, ${ }^{29}$ como uma espécie de retrospectiva por parte do idoso, suas realizações, reconhecimentos e perspectivas em relação ao futuro, e o domínio de intimidade como sentimentos de companheirismo e amor em relação a um companheiro ou pessoa próxima. O estudo citado obteve média para esses domínios de $\geq 15$ pontos e $\geq 14$ pontos respectivamente, o que vai ao encontro com os resultados do presente estudo, que também obteve melhora nos resultados pós intervenção, percebendo assim, pontos positivos refletindo na melhora da qualidade de vida dos idosos da Instituição de Longa Permanência.

A musicoterapia como intervenção em uma instituição de longa permanência, segundo Rocha, ${ }^{30}$ é eficaz para se atingir a melhora da qualidade de vida dos idosos, quanto aos aspectos cognitivos, físicos e emocionais. E deve ser implantada nas propostas de saúde destinadas a população idosa diariamente e não somente através de pesquisas como a do presente estudo. 


\section{CONCLUSÃO}

Com o presente estudo pode-se concluir que a musicoterapia pode ser utilizada como uma ferramenta promotora da estimulação do cognitivo, melhora da qualidade de vida e do fortalecimento da socialização entre os idosos de uma instituição de longa permanência.

Importante ressaltar a vantagem da implementação desta forma terapêutica para o tratamento de idosos, pois os benefícios positivos já demonstrados, podem ser alcançados com um custo reduzido e com uma terapia sem efeitos secundários adversos.

Nota-se então, a necessidade de aplicar estratégias não farmacológicas para amenizar diversas enfermidades e atuar na prevenção através da musicoterapia.

Sendo assim, a música propicia estabelecer um ambiente terapêutico, onde o indivíduo sente-se valorizado e acolhido de uma forma que vai além do sistema convencional de atendimento à saúde.

Como relevância acadêmica, este estudo fortaleceu a pesquisa em saúde do idoso, através de favorecer evidências do instrumento - música, como intervenção e recurso para a reabilitação com um cuidado mais humanizado e holístico de idosos.

Sugere-se ainda que, em estudos futuros, seja aumentado, tanto o tempo de intervenção como o tempo da recolha dos resultados, de forma a perceber realmente o impacto da intervenção ao longo do tempo.

\section{REFERÊNCIAS}

1. World Health Organization. Life in the 21st century: a vision for all. The World Health Report. Geneva: WHO; 1998.

2. Instituto Brasileiro de Geografia e Estatística. Projeção da População 2018: número de habitantes do país deve parar de crescer em 2047 [homepage na internet]. 2018 [acesso em: 07 fev. 2019]. Disponível em:

https://agenciadenoticias.ibge.gov.br/agencia-sala-de-imprensa/2013-agencia-denoticias/releases/21837-projecao-da-populacao-2018-numero-de-habitantes-dopais-deve-parar-de-crescer-em-2047

3. Alves JED. A transição demográfica e a janela de oportunidade [internet]. São Paulo: Instituto Fernand Braudel de Economia Mundial; 2008 [acesso em: 12 fev. 2019]. 13 p. Disponível em:

http://pt.braudel.org.br/pesquisas/arquivos/downloads/a-transicao-demografico-ea-janela-de-oportunidade.pdf 
4. Duca GF, Silva MC, Silva SG, Nahas MV, Hallal PC. Incapacidade funcional em idosos institucionalizados. Rev Bras Ativ Fis Saúde [revista em Internet]. 2011 [acesso em: 12 fev. 2019]; 16(2): 1-5. Disponível em: https://rbafs.org.br/RBAFS/article/view/569

5. Gomes L, Amaral JB. Os Efeitos da Utilização da música para os Idosos: Revisão Sistemática. Revista Enfermagem Contemporânea [revista em Internet]. 2012 dez [acesso em: 12 fev. 2019]; 1(1): 103-117. Disponível em: https://www5.bahiana.edu.br/index.php/enfermagem/article/view/46

6. Guimarães AC, Dutra NS, Silva GLS, Silva MV, Maia, BDC. Atividades grupais com idosos institucionalizados: exercícios físicos funcionais e lúdicos em ação transdisciplinar. Pesquisas e Práticas Psicossociais [revista em Internet]. 2016 jul dez [acesso em: 12 fev. 2019]; 11(2): 443-452. Disponível em: http://pepsic.bvsalud.org/pdf/ppp/v11n2/13.pdf

7. Nunes MI, Ferratti REL, Santos M. Enfermagem em Geriatria e Gerontologia. 1. ed. Rio de Janeiro: Guanabara Koogan; 2012.

8. Barbosa JMM, Prates BSS, Gonçalves CF, Aquino AR, Parentoni AN. Efeito da realização simultânea de tarefas cognitivas e motoras no desempenho funcional de idosos da comunidade. Fisioterapia e Pesquisa [revista em Internet]. 2008 out dez [acesso em: 12 fev. 2019]; 15(4): 374-379. Disponível em: https://www.scielo.br/pdf/fp/v15n4/10.pdf

9. Saldanha LA, Caldas CP. Saúde do idoso AArte de cuidar. 2. ed. Rio de Janeiro: Interciência; 2004.

10. Souza AIN, Ferreira WWAB, Nascimento AG, Silva FFM. A utilização da música como uma terapia para idosos institucionalizados. Anais do IV CIEH; 24-26 set 2015; Campina Grande (PB): Universidade Federal de Campina Grande; 2015.

11. Ribeiro AP, Schutz GE. Reflexões sobre o envelhecimento e bem-estar de idosas institucionalizadas. Rev. Bras. Geriatr. Geronto [revista em Internet]. 2007 mar [acesso em: 12 fev. 2019]; 10(2): 191-201. Disponível em: https://www.redalyc.org/pdf/4038/403838773005.pdf

12. Candeias ARG. Música para a vida: musicoterapia aplicada a idosos institucionalizados. Dissertação. Lisboa: Faculdade de Ciências Humanas e Sociais Instituto de Psicologia e Ciências da Educação, Universidade de Luzíada de Lisboa, 2015.

13. Ministério da Saúde (Brasil). Portaria MS N ${ }^{\circ} 2.528 / 06$. Política Nacional da Saúde da Pessoa Idosa. Brasília, 2006.

14. Gallego MG; García JG. Music therapy and Alzheimer's disease: Cognitive, psychological, and behavioural effects. Neurología [revista em Internet]. 2017 jun [acesso em: 20 nov 2020]; 32(5): 300-308. Disponível em:

https://www.sciencedirect.com/science/article/pii/S217358081730072X 
15. Abrahan VD, Jaramillo AL, Justel N. Benefits of Music Therapy in the Quality of Life of Older Adults. Rev. Cienc. Salud [revista em Internet]. 2019 dez [acesso em: 20 nov 2020]; 17(3): 9-19. Disponível em:

http://www.scielo.org.co/scielo.php?script=sci_arttext\&pid=S1692-

72732019000300009\&lang=pt\#aff1

16. Marques DP. A importância da musicoterapia para o envelhecimento ativo.

Revista Portal de Divulgação [revista em Internet]. 2011 out [acesso em: 26 fev 2019]; (15): 18-24. Disponível em:

https://revistalongeviver.com.br/index.php/revistaportal/article/view/194

17. Seinfeld S, Figueroa H, Ortiz-Gil J, Sanchez-Vives MV. Effects of music learning and piano practice on cognitive function, mood and quality of life in older adults.

Front. Psychol [revista em Internet]. 2013 nov [acesso em: 20 nov 2020]; 810(4): 1-13. Disponível em:

https://www.frontiersin.org/articles/10.3389/fpsyg.2013.00810/full

18. Sárkamo T. Cognitive, emotional, and neural benefits of musical leisure activities in aging and neurological rehabilitation: A critical review. Annals of Physical and Rehabilitation Medicine [revista em Internet]. 2018 nov [acesso em: 20 nov 2020]; 61(6): 414-418. Disponível em:

https://www.sciencedirect.com/science/article/pii/S1877065717300465?via\%3Dih ub

19. Rossetto TCFS. Interface entre a musicoterapia e a terapia ocupacional na estimulação da memória em um grupo de idosos. Monografia. Ribeirão Preto: Universidade de Ribeirão Preto; 2008.

20. Ministério da saúde (Brasil). Concelho Nacional de saúde. Resolução $n^{\circ} 466$, de 12 de dezembro de 2012. Brasília, 2013.

21. Folstein MF, Folstein SE, Mchugh PR. "Mini-mental state". A practical method for grading the cognitive state of patients for the clinician. J Psychiatr Res [revista em Internet]. 1975 nov [acesso em: 17 mar 2019]; 12(3): 189-198. Disponível em: https://www.sciencedirect.com/science/article/abs/pii/0022395675900266

22. Tombaugh TN, Mcintyre MA NJ. The Mini-Mental State Examination: A comprehensive review. Journal of the American Geriatrics Society. Set 1922; 40(9): 922-935.

23. Fleck MP, Chechamovich E, Trentini C. Development and validation of the Portuguese version of the WHOQOL-OLD module. Rev Saúde Pública [revista em Internet]. 2006 set [acesso em: 17 mar 2019]; 40(5):785-791. Disponível em: https://www.scielo.br/pdf/rsp/v40n5/07.pdf

24. Machado JC, Ribeiro RCL, Cotta RMM, Leal PFG. Declínio cognitivo de idosos e sua associação com fatores epidemiológicos em Viçosa, Minas Gerais. Rev. Bras. Geriatr. Gerontol [revista em Internet]. 2011 [acesso em: 12 out 2019]; 14(1): 109-121. Disponível em: https://www.scielo.br/pdf/rbgg/v14n1/a12v14n1 
25. Lourenço RA, Veras RP. Mini-Exame do Estado Mental: características psicométricas em idosos ambulatoriais. Rev Saúde Pública [revista em Internet]. 2006 [acesso em: 12 out 2019]; 40(4): 712-719. Disponível em: https://www.scielo.br/scielo.php?script=sci_arttext\&pid=S003489102006000500023

26. Andrade JFO, Souza LC, Ferrer MLP. Deficiência cognitiva e sua influência sobre os distúrbios de equilíbrio em idosos institucionalizados. FisiSenectus [revista em Internet]. 2017 jan-jun [acesso em: 12 out 2019]; 5(1): 21-30. Disponível em:

https://bell.unochapeco.edu.br/revistas/index.php/fisisenectus/article/view/3680/0

27. Serbim AK, Figueiredo AEPL. Qualidade de vida de idosos em um grupo de convivência. Scientia Medica [revista em Internet]. 2011 [acesso em: 20 out 2019]; 21(4): 166-172. Disponível em:

https://revistaseletronicas.pucrs.br/ojs/index.php/scientiamedica/\%20article/view/ 9405/7236

28. Vieira HVS. Avaliação da qualidade de vida de idosos na universidade aberta à maturidade. Tese. Campina Grande: Universidade Estadual da Paraíba Campus I; 2016.

29. Miranda LC, Banhato EFC. Qualidade de vida na terceira idade: a influência da participação em grupos. Psicologia em Pesquisa [revista em Internet]. 2008 janjun [acesso em: 20 out 2019]; 2(1): 69-80. Disponível em:

http://pepsic.bvsalud.org/pdf/psipesq/v2n1/v2n1a09.pdf

30. Rocha LN. Relevância da musicoterapia na saúde e bem-estar de idosos institucionalizados no município de boa vista-rr. Dissertação. Boa Vista: Universidade Federal de Roraima; 2017.

Artigo recebido em: 04/12/2019

Artigo aprovado em: 03/01/2021

Artigo publicado em: 11/02/2021 